\title{
Perspectivas actuales en la conservación de las ciudades históricas
}

\section{Introducción}

El propósito de este artículo es hablar no sólo de lo que aceptamos específicamente como ciudad histórica en los trabajos de planeamiento especial para la conservación de conjuntos urbanos, sino también de su ampliación a esa otra ciudad que es considerada como algo externo y de la cual se ocupan oficialmente los planes generales o las normas subsidiarias.

También nos proponemos establecer los parámetros en los cuales empieza a desenvolverse la planificación urbana aplicada a los conjuntos históricos, a partir de un factor recurrente, casi obligado, que es la consideración del patrimonio como recurso y la imposibilidad de aislarlo de un contexto mucho más generoso con otros problemas de la ciudad.

\section{La planificación de las ciudades históricas y el municipio}

A partir de la legislación actual en España, cuya matriz inicial es la Ley del Patrimonio Histórico Español de 1985, que en el caso de Andalucía queda apoyada a través de la Ley del Patrimonio Histórico Andaluz de 1991, las actuaciones sobre los Conjuntos Históricos tienen instrumentos específicos como los planes especiales, sin negar el papel que pueden ejercer desde la perspectiva patrimonial otros mecanismos de planificación urbana. Al mismo tiempo se abre la protección del patrimonio a los municipios precisamente a través del planeamiento, estableciendo unas cotas de corresponsabilidad en la gestión con la administración regional competente. Para traducir a la realidad esta orientación, años después de la aparición de la ley, son numerosos los planes especiales donde participan el Ayuntamiento en cuestión y las Consejerías de Cultura y Obras Públicas y Transportes de la Junta de Andalucía, garantizando todos los escalones posibles de las competencias y también del conocimiento de los problemas. Desde el punto de vista de este artículo no interesa tanto analizar la casuística de este hecho positivo de la realidad andaluza, como la constatación de otros problemas que deben interesar por igual a nuestras administraciones locales o regionales y al Estado.

El proceso de planeamiento acaba finalmente en la entrega del instrumento aprobado al Ayuntamiento como institución que tiene encomendada la tarea de administrar la ciudad de forma más directa. Esta lógica impecable desde el punto de vista del procedimiento se estrella de manera estrepitosa en la aplicación y desarrollo de los planes, porque las administraciones locales tienen muchas veces en sus manos planes básicamente bien preparados pero casi ningún medio para cumplir con los objetivos menos rutinarios. De esta forma el aparato de licencias empieza a expedir permisos de obra con la nueva normativa, los agentes implicados en la construcción se felicitan de la ausencia de una regulación engorrosa a través de la Comisión Provincial de Patrimonio Histórico y la ciudad recupera o simplemente sigue con su capacidad de regeneración de tejido, recambio paulatino de población y algún pequeño cambio de perspectiva.

Vista aérea de la Catedral de Cádiz y contexto urbano

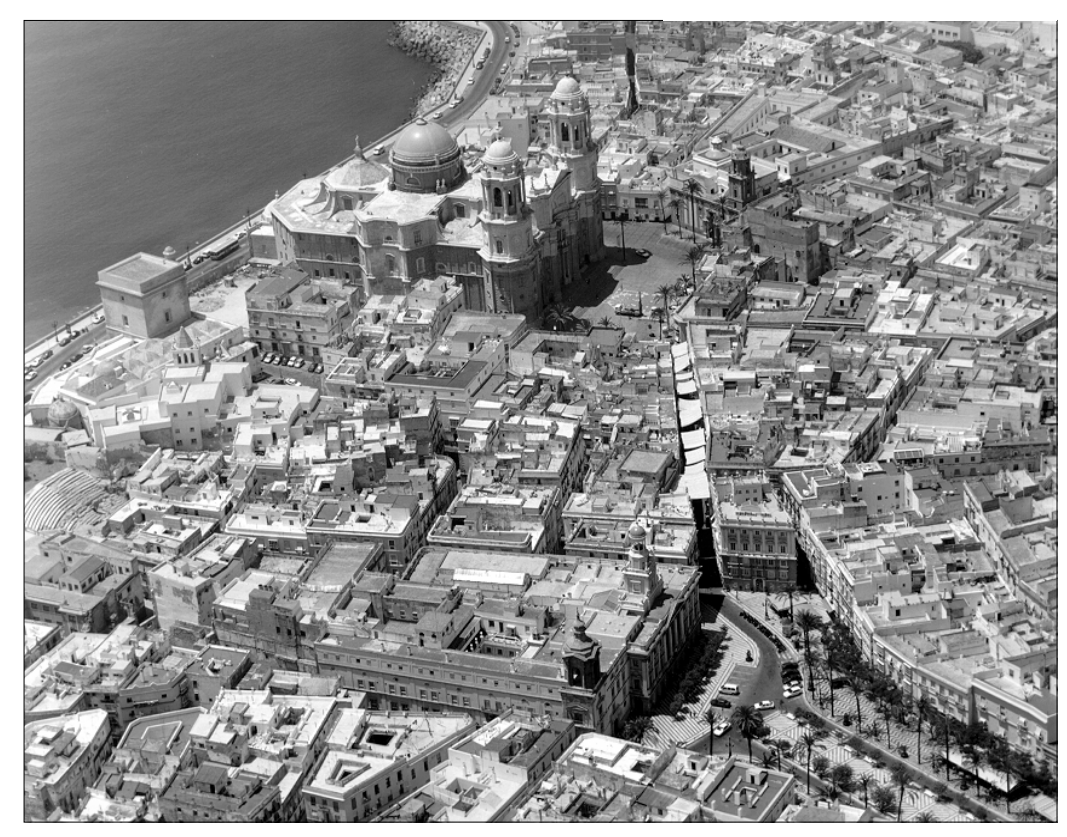

Detrás de gestiones municipales exitosas en el terreno de la conservación de ciudades históricas, se constatan: planes especiales de redacción temprana, largos periodos de estabilidad política, patronatos supramunicipales para concertar voluntades y atraer fondos, empresas municipales o mixtas con participación privada para transformar plusvalías del suelo y ayudas de los fondos europeos etc. Este es el caso de ciudades como Santiago, Gerona, Barcelona, Évora etc. En todos estos ejemplos y otros que pudieran citarse estaremos hablando siempre de estrategias que van más allá de la fe en una normativa urbanística y combinan la tenacidad con la programación de estrategias complejas, muchas de ellas apoyadas en toda la ciudad. 


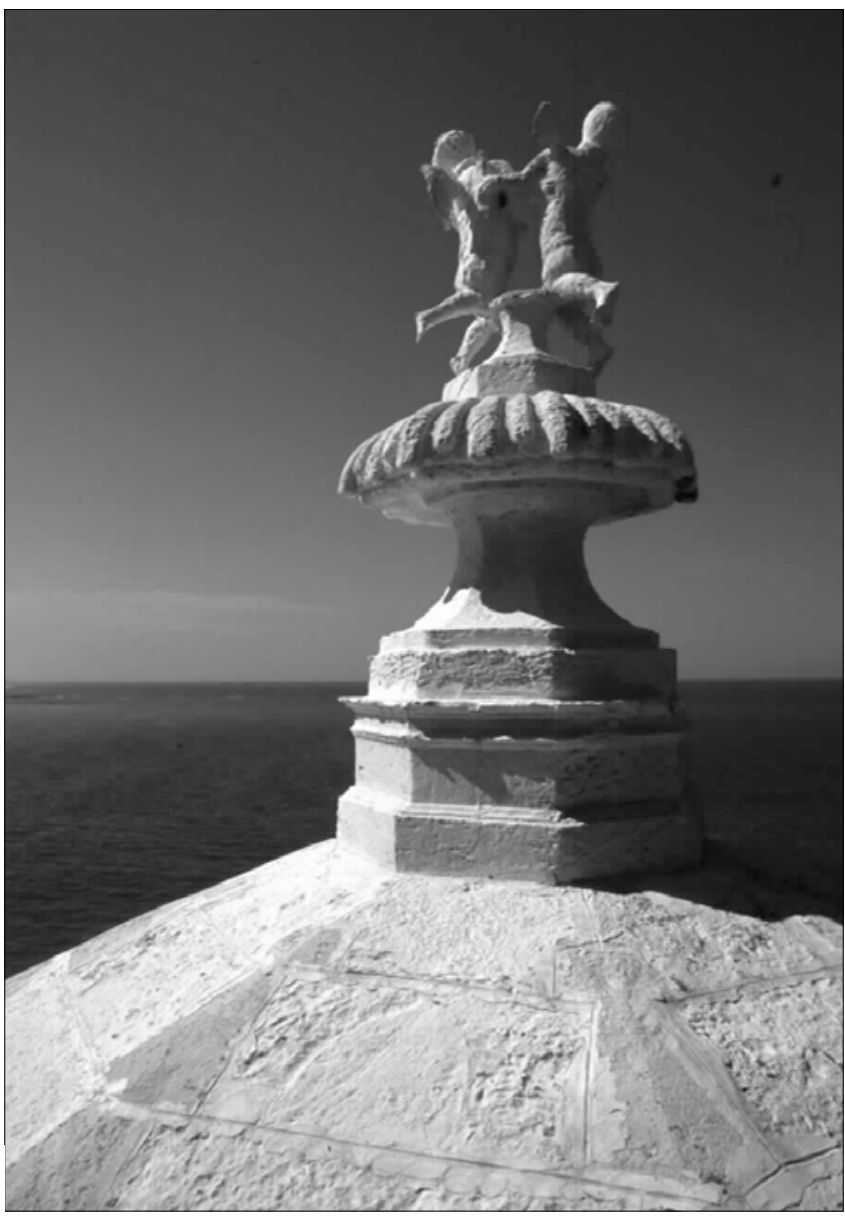

Estas experiencias son fruto de una década de trabajo bajo unas circunstancias favorables que se supieron aprovechar, no es fácil, ni seguramente conveniente, reducir estos tiempos sino aceptar que estas políticas de trenzado complejo requieren un ritmo seguro y que por tanto hay que crear mecanismos de estabilidad en los Ayuntamientos en base a varios principios: a) buenos instrumentos de planificación urbana con contenidos alternativos; b) dotación económica y de recursos humanos para las estrategias que se programen; c) sostenibilidad en el tiempo; d) establecimiento de fuertes vínculos y alianzas entre los partidos, los agentes públicos y privados y los ciudadanos que disuelvan, o al menos amortigüen, las emociones compulsivas de tipo destructivo que se suelen dar tras los recambios electorales.

Como práctica saludable que resume lo anterior resulta preferible no confiar en exclusiva en los medios que puede inyectar la administración para afrontar la ardua y costosa tarea de la rehabilitación en las ciudades históricas porque este exceso de optimismo hace que los procesos sean escasamente sostenibles en el tiempo, se pierde esa continuidad tan necesaria en un campo tan delicado como este, y posiblemente el resultado sea peor ya que los parones en estas políticas equivalen a retrocesos. Las etapas más avanzadas se distinguen por las alianzas en todos los terrenos, tanto en el campo de los agentes como de las acciones, y las administraciones públicas, además de procurar los equilibrios sociales, controlar el proceso y propugnar incentivos, deben mantener una política inversora sostenida en base a unos recursos bien estudiados que permitan la optimización de las infraestructuras urbanas, los espacios públicos y parte de los equipamientos, consiguiendo una mejora paulatina y acompasada de las estructuras físicas y los servicios.

El papel de equilibrio al que hemos hecho referencia por parte de la administración debe ir dirigido en el terreno social a evitar cambios paulatinos de la población residente que es desplazada sistemáticamente, aunque en estos momentos de sofisticación de las políticas de mercado el hecho pase casi inadvertido.

\section{Las fronteras de las políticas sobre patrimonio en la conservación de las ciudades históricas}

La consideración del patrimonio cultural como recurso está obligando a mirar desde otros puntos de vista. Desde la acepción del término bien cultural se viene hablando de la utilización del legado cultural en la planificación económica, añadiendo el término sostenible para no desvincularlo de los mecanismos de protección o de tutela en su sentido más amplio. La cuestión se plantea hace ya dos décadas cuando se advierte la relación tan decisiva entre patrimonio cultural y turismo de masas, siendo esta última una actividad que a finales de los noventa ha adquirido plenamente la categoría de estratégica porque se derivan de ella circunstancias vitales como la participación de la comunicación electrónica, la especialización e intensificación del transporte aéreo, la explosión de los mercados para la ventas de bienes y equipamiento, la transformación de los asentamientos urbanos (hoteles, urbanizaciones e instalaciones de ocio...), la ocupación o reutilización de espacios no urbanizados y un largo etcétera.

La actividad inmobiliaria vinculada con el sector turístico es decisiva y en concreto en España ha protagonizado la transformación del espacio desde finales de los 50 hasta hoy. No es por tanto un aspecto de la cuestión que podamos considerar como nuevo pero sí relacionar claramente la visita a los monumentos, recintos, sitios, lugares etc. como parte de una actividad que no acaba ahí sino que está históricamente especializada en la implicación de múltiples agentes y sectores de la economía y de la industria, que es su vía de penetración natural en los modelos de transformación del espacio.

Lo que se constata es relativamente claro: a medida que la actividad turística despliega su modelo ramificado en la ciudad histórica, los recursos llegan (aunque en menor cantidad de lo que corresponde) a los elementos clave del patrimonio cultural, pero la cohesión del entramado físico y social disminuye. Se trata en buena parte de los casos de un factor de aculturación. Los flujos pueden redistribuirse en el territorio porque cada vez se desplazan más personas y en ese caso el patrimonio cultural menos conocido se convierte en señuelo a través de programas de difusión. 
No sería correcto atribuir al turismo el papel exclusivo en la cadena de acontecimientos que están cambiando la apreciación y valoración del patrimonio cultural pero sí debemos considerar su importante peso en regiones como Andalucía que tienen una actividad hipertrofiada en el sector de los servicios en detrimento de la industria o la investigación científico técnica.

En nuestra opinión no queda otra alternativa que iniciar un debate profundo cuyas consecuencias se inserten en la realidad del ordenamiento urbano y en la planificación estratégica de las ciudades y los territorios de Andalucía y al mismo tiempo abrir esos instrumentos a otras políticas de interés que nos hablen más que nunca de la ciudad y sus habitantes. Porque el pronóstico para muchas de nuestras ciudades, pueblos y territorios, fuertemente implicados con la historia es la intensificación de las tensiones urbanísticas y la constatación de que el abandono a secas es más que nunca la preparación del asedio.

En primer lugar debemos reconsiderar la posición del patrimonio inmaterial que suele deshacerse con más facilidad en actividades que generan monocultivos. La posible permanencia y protagonismo de ciertas tradiciones, oficios, ritos, folclore etc. ya ha sido cuestionada por el proceso de recambio operado en la sociedad española en estas últimas décadas y no puede achacarse su trivialización al fenómeno turístico en exclusiva. Sin embargo, ante la expansión de aquél se están intensificando los procesos de depreciación, incluso a cargo de las propias comunidades locales, cuando existe la alternativa de insertar ese legado en una perspectiva de futuro que le otorgue un nuevo papel y facilite su renovación.

La vivienda para personas de economía modesta o acomodada se resiente en cualquier intensificación de las políticas inversoras. Resulta por un lado necesaria la presencia de agentes privados que promuevan la rehabilitación o la obra nueva en los centros históricos, pero por otro las intervenciones suelen ser bastante drásticas tanto por el tipo de vivienda y de organización espacial proyectadas como por la amplitud de las sustituciones de tipo constructivo y ambiental. El origen del problema está muchas veces en los altos precios de los inmuebles antiguos, en su estado casi ruinoso y en la necesidad de ofrecer al comprador un producto "nuevo dentro de lo antiguo" para avalar el precio final. En este proceso el residente ha quedado descolgado por una ruina previa ganada ante el juzgado o un pacto con una baja indemnización ante la amenaza del desahucio.

Una medida de cierta trascendencia consiste en obstaculizar el abandono de los inmuebles que es la primera etapa que recorre la propiedad. La disciplina vinculada a la declaración de ruina está instaurada en los Ayuntamientos desde hace largo tiempo pero su aplicación es compleja y tiene que moverse entre vericuetos legales largamente conocidos por los propietarios. Solamente se puede abordar el problema con renovados impulsos a partir de dos frentes: la nueva Ley del Suelo de Andalucía que va a crear para los inmuebles mecanismos coercitivos similares a los solares sin edificar, y el Registro de Edificios con Deberes de Conservación de los planes especiales que permitirá a las corporaciones locales aplicar políticas preventivas para impedir o dificultar severamente la ruina técnica y la económica. Se puede constituir como una base de datos que incluya tanto el patrimonio catalogado como el no catalogado que esté en condiciones deficientes, de forma que la ruina no se declare en un proceso finalista e "inesperado". En cualquier caso son necesarios recursos técnicos y humanos suficientes en los ayuntamientos y contar con planes urbanísticos que creen los dispositivos de registro y una normativa con las medidas de conservación pertinentes.

Si el parque inmobiliario cambia en algo tan importante como la velocidad de deterioro habrá más posibilidades de rehabilitaciones "blandas" y de pactos con los vecinos para el acceso diferido a la propiedad a través de los créditos a largo plazo con la intervención puente de las empresas públicas de vivienda y suelo. Desde 1980 hay experiencias muy positivas en acciones de rehabilitación para vivienda propia por parte de la Consejería de Obras Públicas y Transportes en toda Andalucía, quizá haya que completar la aplicación en emplazamientos más complejos y circunstancias especialmente difíciles como las que se dan en algunas ciudades grandes o donde el abandono tiene eficacia probada.

Estas acciones relacionadas con la vivienda son las únicas que en estos momentos se pueden utilizar para conservar la arquitectura vernácula que muchas veces

José Galnares Sagastizábal: Edificio Bruguier y Trujillo en c/ Adolfo Rodríguez Jurado (1938-1940)

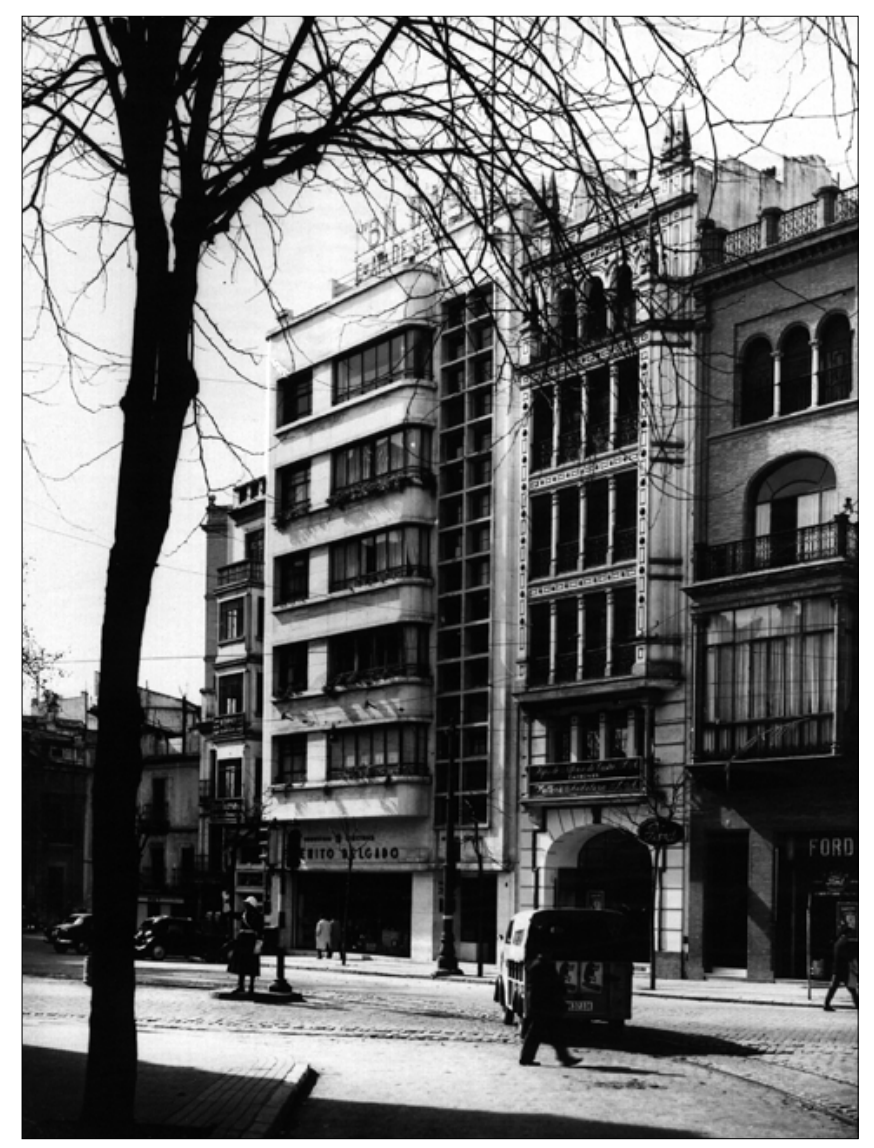




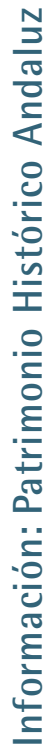

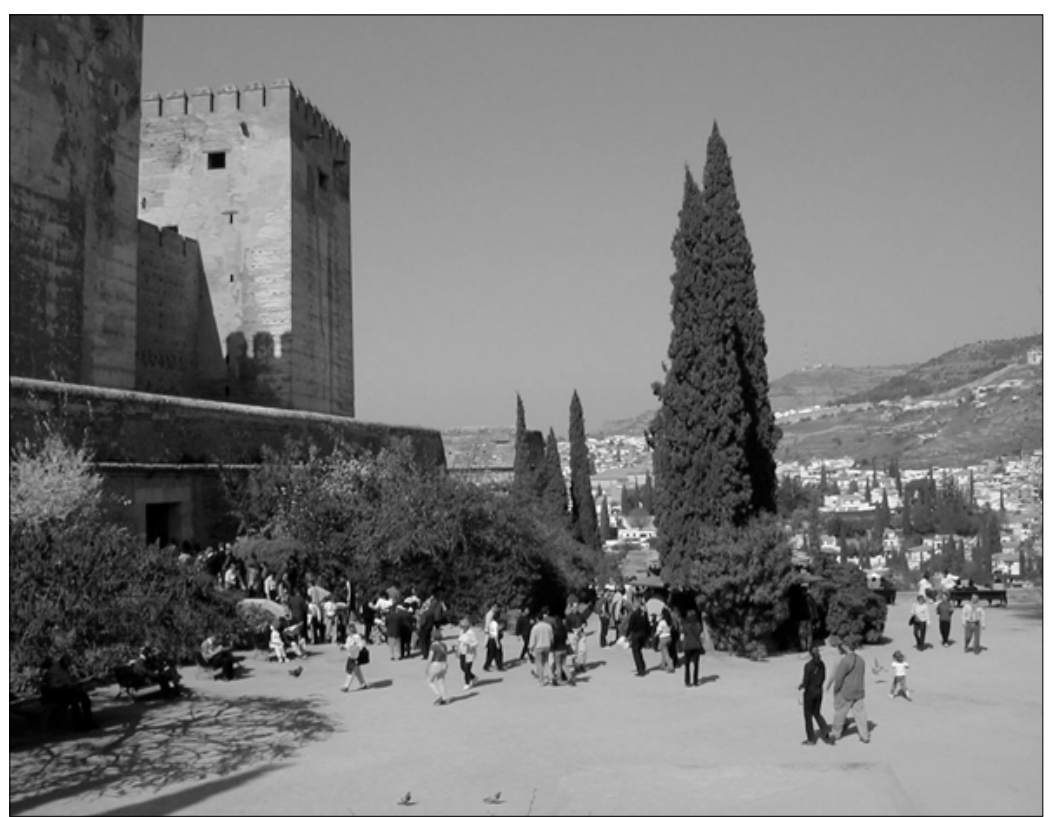

La visita turística y los monumentos: plaza de los aljibes de la Alhambra no llega a los catálogos de los grandes centros históricos pero se considera globalmente como patrimonio a conservar. Generalmente se trata de trama residencial de gran interés donde reside población con nivel económico medio - bajo, arraigada con el lugar, que añade un soporte indispensable al tejido urbano a través de pequeños negocios comerciales y talleres vinculados a oficios de gran interés (carpinteros, ebanistas, herreros, luthiers, etc.).

El propio turismo debe considerarse como una parte de las estrategias de los planes. Un monumento singular como la Alhambra ha dado paso importante en este sentido: su explotación como recurso descansa básicamente sobre el turismo, sin embargo no se sabía cual era la dimensión del problema ni se habían acotado las variables para reconducir sus efectos. Por este motivo el Patronato de la Alhambra y Generalife ha desarrollado un Estudio Previo para la revisión del Plan Especial del conjunto monumental que en buena

parte se ocupa de esta problemática. Los planes de la nueva década deben atender a esta actividad productiva por sus implicaciones en los programas de usos, en el transporte, en el patrimonio cultural, en la ocupación del espacio... En tanto que la ciudad resuelva el conjunto de actividades que tienen lugar, la prestación para el turismo será más eficaz y también más lejana a esas situaciones de especialización crítica: la calle - expositor con camisetas y souvenir, el tranvía con campanita solo para turistas, la ciudad - desierto con locales comerciales de marca y edificios vacíos que se abandona a la caída de la tarde...

Los equipamientos siguen siendo una cuestión pendiente en los cascos antiguos, el abandono progresivo al que están sometidos frente a la política de diversificación funcional de los nuevos barrios, ha dejado en ellos las actividades administrativas fundacionales: probablemente siga allí su Ayuntamiento pero no haya centro de salud o ambulatorio, o centro para la tercera edad o guarderías. La recuperación de la ciudad tendrá que hacerse optando por estos usos y los que ofrezcan posibilidades imaginativas respecto al empleo para completar los que se ofertan desde la actividad terciaria asentada tradicionalmente en el centro.

El otro aspecto a considerar se deriva de las técnicas de intervención. De los sistemas tradicionales queda mucho que aprender, de hecho los revestimientos derivados de la cal y los pigmentos naturales constituyen un frente de gran interés reabierto con éxito en algunas zonas donde existen aplicadores con conocimientos suficientes. Las posibilidades plásticas de estas técnicas están sirviendo para conseguir excelentes resultados también en la obra nueva. Como alternativa a las cartas comerciales de colores montadas sobre pintura acrílica es preferible recuperar plenamente este tipo de revestimientos, ofrecer algo más que una gama de colores para entrar en propuestas de acabados y texturas y fomentar una industria de la construcción local o regional que atienda a una demanda que en estos momentos está creciendo de manera clara en muchas ciudades. Actualmente estas propuestas empiezan a tener eco en muchas industrias que están diversificando productos en el ramo de la cerámica (ladrillos especiales, pavimentos y azulejería) y los morteros preparados. Es una cuestión de detalle que al final se convierte en la gran protagonista porque las intervenciones se suceden en los cascos antiguos, en un momento al alza de la actividad constructora, pero los resultados están transformando los ambientes en algo tan esencial como el color y los acabados, cuestión a resolver por medios alternativos a la normativa, es decir aportando conocimiento, maestrías y materiales aptos.

Nos encontramos de nuevo con el título del epígrafe, las condiciones de conservación de las ciudades habrá que buscarlas en estos y otros campos cuyas fronteras están aún por definir, intentando pautas que abarquen otras disciplinas capaces de sumarse a las ya tradicionales en el planeamiento urbano, que debe convertirse sin cortapisas en un lugar de confluencia.

\section{La nueva ciudad}

Frente a la preocupación sobre la conservación de ciudades que ha hecho derramar ríos de tinta y pocas acciones coherentes en los cascos antiguos, o en esa parte de ciudad que en cada sitio se reconoce como histórica, hay que reclamar otros supuestos que van más allá de la ciudad histórica como hecho aislado y rescatan el interés hacia otras arquitecturas y otras tramas.

La arquitectura moderna que se desarrolla en Europa a partir de la Gran Guerra crea un sustrato con una influencia decisiva para el futuro. Se sientan las bases para los nuevos ensanches, se codifica una estética diferente y se anticipa la capacidad de acción que se demandará en las grandes reconstrucciones que vendrán después de la Segunda Guerra Mundial. No todo lo 
que se construye es considerado arquitectura moderna, no todo lo que se hace puede medirse con el mismo fiel aunque sea contemporáneo, pero esto también es parte de su herencia.

Las propuestas estéticas de los nuevos maestros, las directrices expuestas en textos considerados revolucionarios en el orden urbano, permiten experimentos de gran interés que conviven con todo tipo de arquitecturas y ciudades. Lo que es más radical desde una visión plena de lo moderno tiene lugar en nuestro país en lugares como el País Vasco o Cataluña más cercanos a la experiencia europea, en otras zonas los experimentos son más aislados y será una posguerra distinta a la del resto de Europa la que acabe rizando el rizo para jugar entre la modernidad y el regionalismo.

En estos últimos años un esfuerzo de gran interés, el DOCOMOMO, Documentación y Conservación de la Arquitectura y el Urbanismo del Movimiento Moderno, logra a través de sus seminarios y publicaciones reunir y dar coherencia a lo disperso y mal conocido, para reclamar el interés extraordinario de otra forma de hacer ciudad.

El interés de estos trabajos debe ir lógicamente más allá de una trasposición de estas presencias a un público especializado, la propuesta sería utilizar estas aportaciones para devolver una nueva mirada a toda la ciudad, logrando romper escisiones a partir de la historia que han sido no sólo una cuestión de convenciones sino también de procedimiento y de exclusión.

Esta actitud menos maniqueísta permitiría también la consideración de otras arquitecturas que constituyen el sustrato de muchos pueblos y ciudades y que bajo la adscripción de patrimonio menor están siendo destruidas o arrinconadas sistemáticamente sin que se presenten alternativas de interés.

Al mismo tiempo la arquitectura de hoy debe formar parte de las ciudades antiguas permitiendo sustituciones con nuevos códigos estéticos, esta compatibilidad se ha mostrado rentable en otras épocas a través de excelentes ejemplos que no han seguido comportamientos miméticos.

Para entender este modelo alternativo en la conservación de las ciudades históricas es imprescindible que los esfuerzos realizados desde DOCOMOMO se materialicen en las formas de planificar y en los instrumentos de protección que creen las administraciones públicas. Esta es justamente la llamada de atención que hace el grupo DOCOMOMO Ibérico en el Manifiesto de Zaragoza del 13 de noviembre de 1997, tras el primer seminario que se desarrolló en dicha ciudad, recordando ejemplos flagrantes de abandono de arquitecturas del siglo $X X$ cuya pérdida no deberían permitir países como España y Portugal.

\section{Bibliografía}

AAVV. La habitación y la ciudad modernas: rupturas y continuidades. 1925-1965. Primer Seminario DOCOMOMO Ibérico, Zaragoza 13-15 de noviembre de 1997.

Rojas, E y Moura de Castro C. Préstamos para la conservación del Patrimonio histórico Urbano. Serie de informes técnicos del Depar- tamento de Desarrollo sostenible. Banco Interamericano de Desarrollo. Washington D. C., 1999.

Troitiño, M. A. y cols. Estudio Previo para la Revisión del Plan Especial de la Alhambra y Alijares. Patronato de la Alhambra y Generalife. Granada, 1999. 